\title{
Evaluating Effectiveness of Medical Grade Honey-Containing Alginate Dressing in Patients with Chronic Lower Extremity Wounds
}

\author{
Yeon Ji Lee (D), Kyeong Soo Park (D), Dong Yeon Kim (D), Hyung-Sup Shim (D) \\ Department of Plastic and Reconstructive Surgery, St. Vincent's Hospital, College of Medicine, The Catholic University of Korea, Suwon, Korea
}

\begin{abstract}
Background: Chronic wounds of the lower extremities are challenging to treat. Recently, honey-based dressings have been shown beneficial effects in diabetic foot ulcers and venous ulcers. Here, we compared Medihoney alginate with a standard alginate dressing in lower extremity chronic wounds.

Methods: We performed retrospective chart reviews of 37 patients between January 2019 and January 2021 with chronic lower extremity wounds who were treated with an Algisite M dressing (group A) or a Medihoney alginate dressing (group B). Microorganisms isolated from wound cultures, weekly decrease in wound area over 4 weeks, the number of patients who achieved complete wound closure, and the amount of time required for wound closure were compared between the two groups.

Results: Sixteen patients were assigned to group A and 21 to group B. The isolated microorganisms were confirmed as methicillin-susceptible Staphylococcus aureus most commonly, followed by methicillin-resistant $S$. aureus. Wound area decreased from 12.71 to $2.34 \mathrm{~cm}^{2}$ in group $A$ and from 14.07 to $1.71 \mathrm{~cm}^{2}$ in group $B(P<0.05)$. Seven patients achieved complete wound closure in group $A(43.75 \%)$ and 15 patients in group $B(71.43 \%)$. Mean duration of wound closure was 36.31 days in group A and 34.62 days in group $B$.

Conclusion: Considering the more rapid decrease in wound area in group B, application of a honey-containing alginate dressing to lower extremity chronic wounds, especially those infected with antibiotic-resistant bacteria, was proved to be a good treatment option.
\end{abstract}

Keywords: Diabetic foot ulcers; Venous ulcers; Pressure sore; Honey; Alginate

\section{Introduction}

Chronic wounds are defined as wounds that have not healed after 3 months of treatment [1]. Chronic wounds are mainly caused by bacterial biofilm formation and are increasingly being caused by antibiotic-resistant bacteria [2]. Chronic wounds in the lower extremities are considered more challenging to treat than wounds in other areas of the body due to decreased blood flow in the lower extremities. Diabetic foot ulcers (DFUs), venous stasis ulcers, and chronic ischemic wounds resulting from vascular disease are common examples of chronic lower extremity wounds [3]. Various dressing materials and techniques have been explored for treating lower extremity chronic wounds [4]. Honey-based dressing materials in particular have been proposed as good potential dressing options [5]. Although wounds have been treated with honey in various cultures for thousands of years, scientific evidence of honey's effectiveness has only recently been provided [6]. Honey has been reported to inhibit bacterial growth, prevent biofilm formation, and remove biofilm when applied to chronic wounds [7]. In recent years, honey-based dressings have been shown to have beneficial effects when used to treat DFUs, venous stasis ulcers, and pressure sores

\section{Original Article}

Received: March 2, 2021

Revised: July 21, 2021

Accepted: July 22, 2021

Corresponding author: Hyung-Sup Shim, M.D., Ph.D.

Department of Plastic and Reconstructive Surgery,

St. Vincent's Hospital, College of Medicine, The

Catholic University of Korea, 93 Jungbu-daero,

Paldal-gu, Suwon 16247, Korea

Tel: +82-31-249-7206

Fax: +82-31-241-0005

E-mail: sharpshim@catholic.ac.kr

This is an Open Access article distributed under the terms of the Creative Commons Attribution Non-Commercial License (https://creativecommons.org/licenses/by-nc/4.0/) which permits unrestricted non-commercial use, distribution, and reproduction in any medium, provided the original work is properly cited.

(c) 2021 Korean Wound Management Society 
$[5,8,9]$.

However, the effectiveness of honey-containing alginate dressing materials for the management of chronic wounds of the lower extremities have not been reported. In this study, we compared the wound healing effects of a medical grade honey-containing alginate dressing to a standard alginate dressing.

\section{Methods}

\section{Ethics statement}

This study was approved by the Institutional Review Board of Human Research Protection Program (IRB No. VC20RASI0224). All data were analyzed anonymously and according to the principles outlined in the Declaration of Helsinki 1975 (revised in 2008). All patients provided informed consent for the publication of this study.

\section{Patients}

Retrospective chart review was conducted on patients seen from January 2019 to January 2021 who were treated for chronic wounds on their lower extremities in our department. Most of the patients with chronic wounds on their lower extremities were referred to our department with failed wound closure despite more than 3 months of wound care at other hospitals. Among those patients, those treated with alginate dressing (Algisite M; Smith \& Nephew, Hull, UK), or honeycontaining alginate dressing (Medihoney alginate; Derma Sciences Inc., Princeton, NJ, USA) were included in this study. Demographic information collected included age, sex, type of chronic wounds, location of wounds, and number of wounds. Assessment of initial wound condition included size of the wound surface, depth including exposed level, degree of exudate, and character of the exudate. Microorganisms cultured from the wound were recorded. Wounds were usually classified as dry, moist, wet, saturated, or leaking based on the amount of exudate using the World Union of Wound Healing Societies (WUWHS) criteria (2007) [10].

Based on the type of dressing used, patients were divided into two groups. Patient whose wounds were treated with alginate dressing (Algisite M; Smith \& Nephew) were assigned to group $\mathrm{A}$, and those treated with honey-containing alginate were assigned to group B. Dressing material used in group B was Medihoney alginate containing medical grade Manuka honey. In both groups A and B, superabsorbent polymer (DryMax Foam; Absorbent, Kista, Sweden) was applied as the secondary dressing for effective exudate absorption to prevent in- fection and promote wound healing.

As additional treatment after applying dressing materials, elastic bandage compression was applied to all venous ulcer patients, and $0.12 \mathrm{mg}$ of beraprost sodium (Berasil; Astellas Pharma Inc., São Paulo, Brazil) was applied as daily medication to all diabetes foot ulcer patients according to our routine treatment protocol. There was no difference between the two groups in patient assignment.

Wound beds of most of the chronic wounds were surgically debrided before the dressing was applied. Dressing changes were usually performed 2-3 times a week in the first week, and 1-2 times a week from the second week onwards, but when dressing changes were performed varied depending on the wound condition.

Empirical oral antibiotics therapy was administrated at the first visit in most patients and modified with selective oral antibiotics according to tissue culture results. The duration of antibiotics administration was determined by wound condition.

The number of patients who achieved complete wound closure during 4 weeks of follow-up and the duration until complete wound closure was achieved were assessed in each group. The percentage decrease in wound area every week was calculated by applying the following formula:

Percentage decrease in wound area $(\%)=$

$\frac{\text { (Initial wound area-observed wound area) }}{\text { (Initial wound area) }} \times 100$

Sizes of all wounds were measured using ImageJ software (National Institutes of Health, Bethesda, MD, USA) [11].

\section{Statistical analysis}

Patient demographics including age, sex, the type of chronic wound, location of wounds, and number of wounds were compared between groups using the Mann-Whitney $U$ test and Fisher extraction.

Initial characteristics of the chronic wounds including initial area, depth, degree of exudate, and isolated microorganism by culturing were analyzed and compared using the Mann-Whitney test and Fisher exact test.

The decrease in wound area every week was compared between groups using repeated-measures analysis of variance. The number of patients who achieved complete wound closure within 4 weeks was compared between groups using Fisher exact test, and the duration of complete wound closure was compared between groups using the Mann-Whitney $\mathrm{U}$ 
test. All statistical analyses were executed in GraphPad Prism version 9.0.1 (GraphPad Software Inc., San Diego, CA, USA). To reduce the conditional probability of assignment to a specific treatment group by effects of covariates, the propensity scores were estimated using logistic regression and matched by nearest neighbor matching method [12]. The co-variants include age, sex, the type of chronic wound, location of wounds, number of wounds, depth of wounds, degree of exudate, and, isolated microorganism by culturing.

\section{Results}

From January 2019 to January 2021, a total of 82 patients presented to our department with chronic wounds of the lower extremities. Sixteen of these patients were treated with Algisite $\mathrm{M}$ and DryMax Foam dressing (6 males, 10 females) and assigned to group A, and 21 patients were treated with Medihoney alginate and DryMax Foam dressing (11 males, 10 females) and assigned to group $B$.

Mean patient age was similar between groups $(61.13 \pm 12.62$ years in group $A$ and $58.95 \pm 13.51$ years in group $B)(P>0.05)$.
Diabetic wounds were the most common, occurring in 24 patients $(64.86 \%)$, followed by venous stasis ulcers in eight patients (21.62\%) and pressure sores in five patients (13.51\%) (Table 1). In the location of wounds, foot area was most common, occurring in 24 patients (64.86\%), followed by pretibial area in nine patients $(24.32 \%)(\mathrm{P}>0.05)$. In the number of wounds, single wound was the most common, occurring in 22 (59.46\%) and there was no difference between the two groups $(\mathrm{P}>0.05)$ (Table 1).

Mean area of the initial wound was $12.71 \mathrm{~cm}^{2}( \pm 3.12)$ in group A and $13.89 \mathrm{~cm}^{2}( \pm 3.25)$ in group $\mathrm{B}$, which was not a significant difference. Initial depth of the wound was most commonly dermal to subcutaneous in nine patients (56.25\%) in group A and 13 patients (61.90\%) in group B. Most wounds were classified as being "saturated" in both groups based on the amount of exudate, followed by "leaking." Methicillin-susceptible Staphylococcus aureus was the most common microbe isolated; it was found in the wounds of six patients (37.50\%) in group A and seven patients (33.33\%) in group B, followed by methicillin-resistant $S$. aureus in three patient (18.75\%) in group A and five patients (23.80\%) in group B,

Table 1. Patient demographics

\begin{tabular}{|c|c|c|c|c|}
\hline Variable & $\begin{array}{c}\text { Total } \\
(n=37)\end{array}$ & $\begin{array}{l}\text { Algisite } M \\
\text { dressing group } \\
(n=16)\end{array}$ & $\begin{array}{l}\text { Medihoney alginate } \\
\text { dressing group } \\
(n=21)\end{array}$ & P-value ${ }^{a)}$ \\
\hline Age (yr) & $59.89 \pm 13.18$ & $61.13 \pm 12.62$ & $58.95 \pm 13.51$ & 0.631 \\
\hline Sex & & & & 0.509 \\
\hline Male & $17(45.94)$ & $6(37.50)$ & $11(52.38)$ & \\
\hline Female & $20(54.05)$ & $10(62.50)$ & $10(47.62)$ & \\
\hline Cause of chronic wounds & & & & 0.165 \\
\hline Diabetes foot ulcer & $24(64.86)$ & $8(50.00)$ & $16(76.19)$ & \\
\hline Venous ulcer & 8 (21.62) & $4(25.00)$ & 4 (19.05) & \\
\hline Pressure sore & $5(13.51)$ & $4(25.00)$ & $1(4.76)$ & \\
\hline Location of wounds & & & & 0.544 \\
\hline Pretibial area & 9 (24.32) & $5(31.25)$ & $4(19.04)$ & \\
\hline Calf area & $3(8.11)$ & $2(12.50)$ & $1(4.76)$ & \\
\hline Ankle area & $4(10.81)$ & $2(12.50)$ & $2(9.52)$ & \\
\hline Foot & $21(56.75)$ & $7(43.75)$ & $14(66.67)$ & \\
\hline No. of wounds & & & & 0.145 \\
\hline 1 & $22(59.46)$ & $9(56.25)$ & $13(61.90)$ & \\
\hline 2 & $11(29.73)$ & $5(31.25)$ & $6(28.57)$ & \\
\hline More than 3 (multiple) & $4(10.81)$ & $2(12.50)$ & $2(9.52)$ & \\
\hline
\end{tabular}

Values are presented as mean \pm SD or number (\%).

a) Statistically significant, $\mathrm{P}<0.05$. 
Pseudomonas aeruginosa in three patients (18.75\%) in group A and four patients (19.04\%) in group B, and Acinetobacter in one patient (6.25\%) in group A and two patients (9.52\%) in group B. Multiple organisms were cultured from the chronic wounds of three patients (18.75\%) in group A and three patients (14.28\%) in group B. There with no significant differences in the microbial species cultured from the wounds between the two groups (Table 2).

Wound area decreased from $12.71 \pm 3.97$ to $2.34 \pm 1.30 \mathrm{~cm}^{2}$ in group $\mathrm{A}$, and from $14.07 \pm 3.14$ to $1.71 \pm 1.21 \mathrm{~cm}^{2}$ in group $\mathrm{B}$ by 4 weeks. On a weekly basis, wound area decreased from $12.71 \pm 3.97$ to $8.10 \pm 2.00 \mathrm{~cm}^{2}(36.13 \% \pm 6.87 \%)$ by the 1 st week, $5.80 \pm 2.53 \mathrm{~cm}^{2}(55.19 \% \pm 8.94 \%)$ by the $2 \mathrm{nd}$ week, $3.99 \pm 1.88$ $\mathrm{cm}^{2}(69.28 \% \pm 10.26 \%)$ by the $3 \mathrm{rd}$ week, and $2.34 \pm 1.30 \mathrm{~cm}^{2}$ $(82.26 \% \pm 8.87 \%)$ by the 4 th week in group A. In group B, wound area decreased from $14.07 \pm 3.14$ to $7.33 \pm 2.13 \mathrm{~cm}^{2}(47.54 \% \pm$ $9.72 \%)$ by the 1 st week, $4.69 \pm 1.89 \mathrm{~cm}^{2}(67.40 \% \pm 9.66 \%)$ by the 2nd week, $2.89 \pm 1.59 \mathrm{~cm}^{2}(80.39 \% \pm 8.04 \%)$ by the $3 \mathrm{rd}$ week, and $1.71 \pm 1.21 \mathrm{~cm}^{2}(88.34 \% \pm 7.78 \%)$ by the 4 th week.
The decrease in wound area was greater in group B than in group $\mathrm{A}$. Wound healing was accelerated in group $\mathrm{B}$ compared to group A especially during the first week based on the decrease in wound area $(\mathrm{P}<0.05)$ (Fig. 1). The percentage decrease in wound area was $82.26 \% \pm 8.87 \%$ in group A and $88.34 \% \pm 7.78 \%$ in group $\mathrm{B}$ by the end of the 4 weeks $(\mathrm{P}<0.05)$ (Fig. 1B).

After the propensity score matching by nearest neighbor matching method, 13 pairs of patients from each group were reevaluated resulting that reduction of wound area was greater in group $\mathrm{B}$ than in group $\mathrm{A}(\mathrm{P}=0.021)$ (Supplementary Figs. 1, 2).

Seven of 16 patients achieved complete wound closure in group A (43.75\%) and 15 of 21 patients achieved complete wound closure in group B (71.43\%) by 4 weeks. Mean duration of wound closure was $36.31 \pm 11.12$ days in group $A$ and $34.62 \pm 14.31$ days in group B $(\mathrm{P}>0.05)$ (Table 3$)$. The clinical application of medihoney alginate on diabetic foot and venous ulcer was presented in Figs. 2 and 3, resulting in complete wound healing after 4 weeks.

Table 2. Initial wound status

\begin{tabular}{|c|c|c|c|}
\hline Variable & $\begin{array}{l}\text { Algisite } M \text { dressing group } \\
\qquad(n=16)\end{array}$ & $\begin{array}{l}\text { Medihoney alginate dressing group } \\
\qquad(n=21)\end{array}$ & P-value \\
\hline Initial wound size $\left(\mathrm{cm}^{2}\right)$ & $12.71 \pm 3.12$ & $13.89 \pm 3.25$ & 0.251 \\
\hline Depth of initial wounds & & & 0.749 \\
\hline Dermal to subcutaneous exposure level & $9(56.25)$ & $13(61.90)$ & \\
\hline Muscle exposure level & $7(43.75)$ & $8(38.10)$ & \\
\hline \multicolumn{4}{|l|}{ Exudate } \\
\hline Type & & & $>0.999$ \\
\hline Watery & $10(62.50)$ & $12(57.14)$ & \\
\hline Thick & $6(37.50)$ & $9(42.28)$ & \\
\hline Level & & & 0.899 \\
\hline Dry & $2(12.50)$ & $1(4.76)$ & \\
\hline Moist & $2(12.50)$ & 4 (19.05) & \\
\hline Saturated & $8(50.00)$ & $10(47.62)$ & \\
\hline Leaking & $4(25.00)$ & $6(28.57)$ & \\
\hline Bacteria isolated by culturing & & & 0.998 \\
\hline MSSA & $6(37.50)$ & 7 (33.33) & \\
\hline MRSA & $3(18.75)$ & $5(23.80)$ & \\
\hline Pseudomonas aeruginosa & $3(18.75)$ & $4(19.04)$ & \\
\hline Acinetobacter baumannii & $1(6.25)$ & $2(9.52)$ & \\
\hline Multiple mixed organism & $3(18.75)$ & $3(14.28)$ & \\
\hline
\end{tabular}

Values are presented as mean \pm SD or number (\%).

MSSA, methicillin-susceptible Staphylococcus aureus; MRSA, methicillin-resistant $S$. aureus.

a) Statistically significant, $\mathrm{P}<0.05$. 

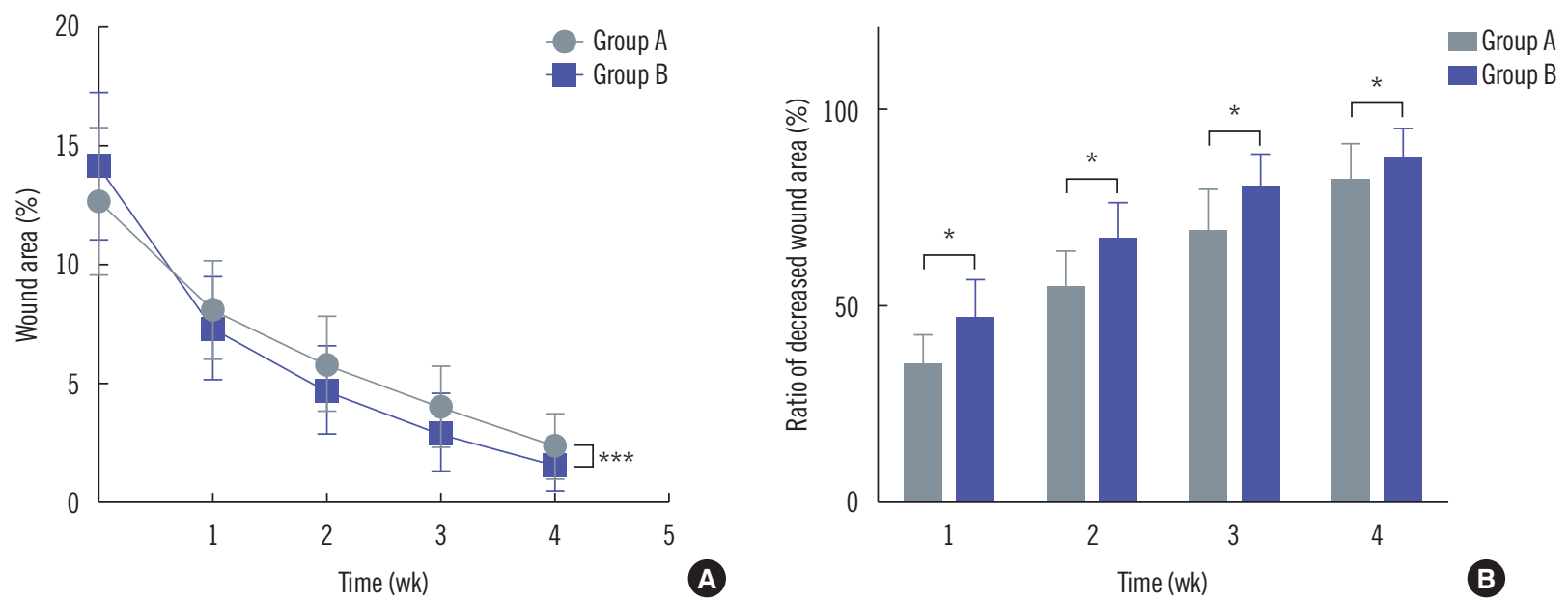

Fig. 1. Decrease in wound area over 4 weeks. (A) Wound area decreased from $12.71 \pm 3.97$ to $2.34 \pm 1.30 \mathrm{~cm}^{2}$ in group A and from $14.07 \pm 3.14$ to $1.71 \pm 1.21 \mathrm{~cm}^{2}$ in group B by 4 weeks $(P<0.001)$. (B) Decrease in percentage wound area was $82.26 \% \pm 8.87 \%$ in group $A$ and $88.34 \% \pm 7.78 \%$ in group $B$ by 4 weeks $(P<0.05)$. The decrease in wound area was faster in group $B$ than group $A$ especially during the first week after treatment. Significant differences were evaluated by repeated-measures analysis of variance. Group A, Algisite M dressing group; group B, Medihoney alginate dressing group.

Table 3. Decrease in wound area over 4 weeks

\begin{tabular}{|c|c|c|c|c|}
\hline Variable & $\begin{array}{l}\text { Algisite } M \text { dressing } \\
\text { group }(n=16)\end{array}$ & $\begin{array}{l}\text { Medihoney alginate dressing } \\
\text { group }(n=21)\end{array}$ & P-value & P-value (PSM) \\
\hline Decreased in wound area & & & $<0.001^{\text {b) }}$ & $0.021^{\text {a) }}$ \\
\hline Initial area $\left(\mathrm{cm}^{2}\right)$ & $12.71 \pm 3.97$ & $14.07 \pm 3.14$ & & \\
\hline 1st week & $8.10 \pm 2.00$ & $7.33 \pm 2.13$ & & \\
\hline 2nd week & $5.80 \pm 2.53$ & $4.69 \pm 1.89$ & & \\
\hline 3rd week & $3.99 \pm 1.88$ & $2.89 \pm 1.59$ & & \\
\hline 4th week & $2.34 \pm 1.30$ & $1.71 \pm 1.21$ & & \\
\hline Decrease in wound area (\%) & & & $0.023^{a)}$ & \\
\hline Initial to 1st week & $36.13 \pm 6.87$ & $47.54 \pm 9.72$ & & \\
\hline Initial to 2nd week & $55.19 \pm 8.94$ & $67.40 \pm 9.66$ & & \\
\hline Initial to 3rd week & $69.28 \pm 10.26$ & $80.39 \pm 8.04$ & & \\
\hline Initial to 4th week & $82.26 \pm 8.87$ & $88.34 \pm 7.78$ & & \\
\hline Patients with complete wound closure by 4 weeks & $7(43.75)$ & $15(71.43)$ & 0.107 & \\
\hline Duration to wound closure (day) & $36.31 \pm 11.12$ & $34.62 \pm 14.31$ & 0.705 & \\
\hline
\end{tabular}

Values are presented as mean $\pm S D$ or number $(\%)$.

PSM, propensity score matching.

Statistically significant, ${ }^{\text {al } P}<0.05,{ }^{\text {bl } P}<0.001$.

\section{Discussion}

Alginate dressing materials are widely used to treat wounds, especially contaminated wounds with a large amount of exudate, as they can reduce microbial burden, absorb exudate, and have excellent biocompatibility [13]. Alginates (algin) are linear anionic polysaccharide polymers of $\beta-(1-4)$-D- mannuronic (M-blocks) and a-L-guluronic acid (G-blocks) extracted from brown seaweed (macroalgae) that contain a large amount of alginic acid [14]. In recent randomized controlled trials, alginate dressing materials were shown to have significant beneficial effects when applied to lower extremity chronic wounds such as venous leg ulcers [15] and DFUs [16]. One of the advantages of alginate as a biocompatible biopoly- 

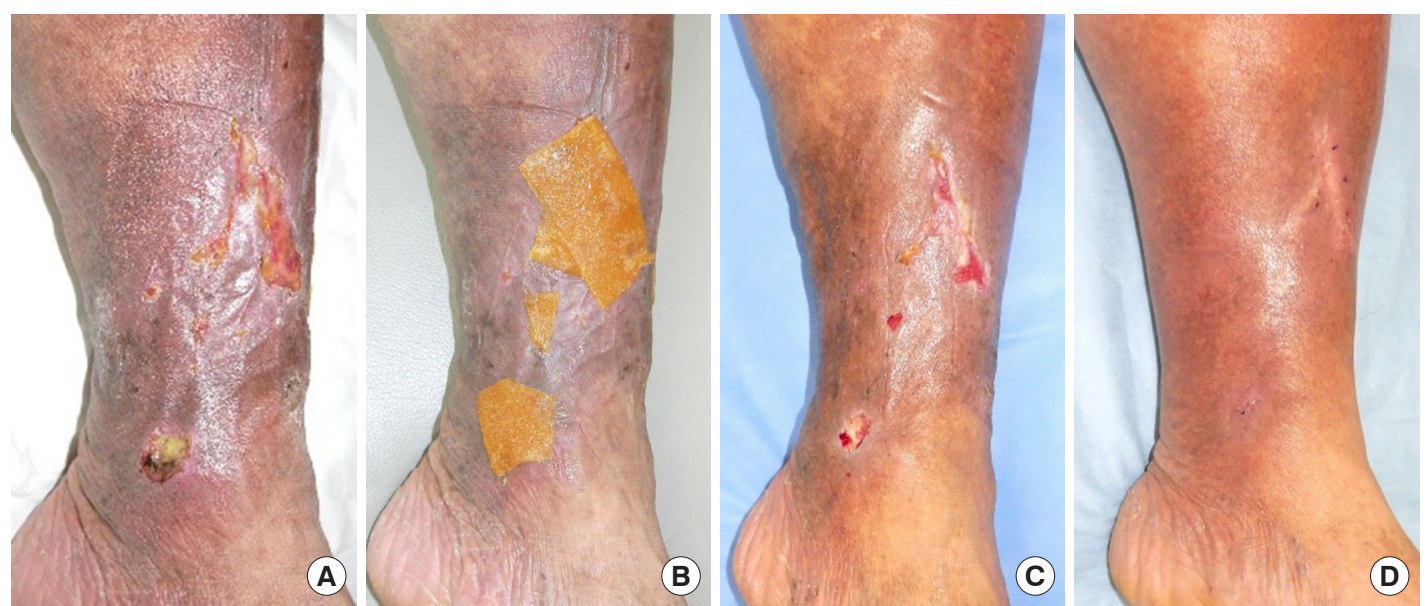

Fig. 2. The application of Medihoney alginate on chronic lower extremity wounds. A 71-year-old female patient with diabetes had calf injury due to trauma. (A) Clinical photo showing a skin defect of about $5.14 \mathrm{~cm}^{2}$ on the anterior portion of the calf. (B) Honey-containing alginate dressing was used to treat the wound. (C) The clinical photo taken about 1 week later shows that the size of the wound had decreased to about $1.32 \mathrm{~cm}^{2}$. (D) Picture obtained 3 weeks after treatment showing complete wound closure.
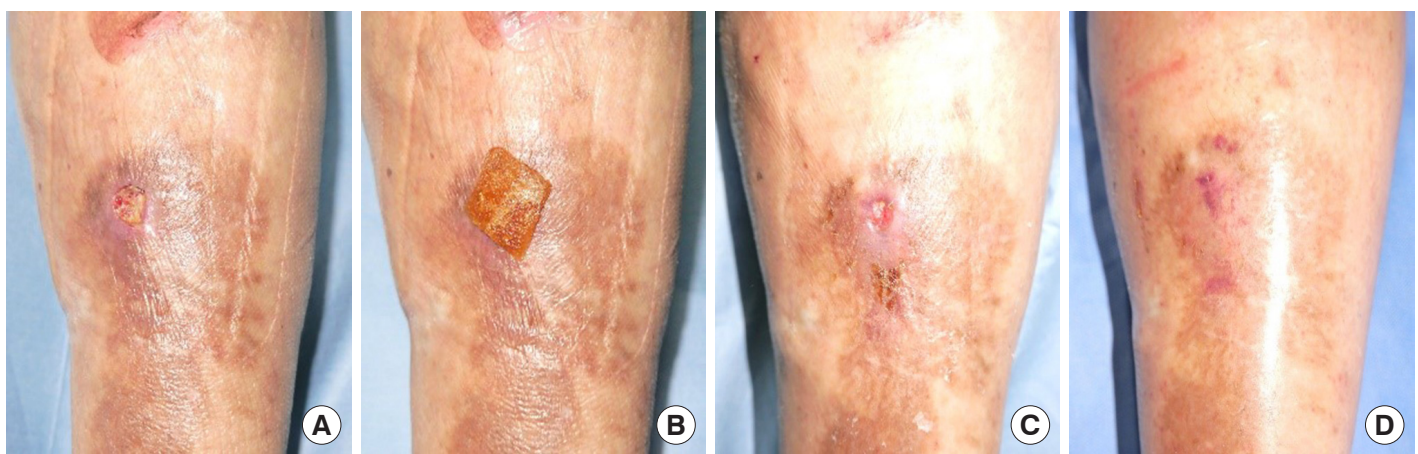

Fig. 3. The application of Medihoney alginate on venous ulcers. A 68-year-old male patient with a history of venous stasis ulcers developed a chronic ulcer in his calf after cellulitis. (A) Clinical photo shows a skin defect on the medial portion of the calf of about $11.25 \mathrm{~cm}^{2}$. (B) Honey-containing alginate dressing was used to treat the wound. (C) The clinical photo taken approximately 2 weeks after treatment showed that the wound had decreased in size to about $6.57 \mathrm{~cm}^{2}$. (D) After 4 weeks of wound care, the wound had healed completely.

mer is that by combining it with various other dressings, synergic effects can be obtained, which can promote healing of complex chronic wounds [13]. The beneficial effects of an alginate-based honey hydrogel film for the treatment of complex infected wounds was demonstrated in an in vivo study using an infected burned rat model [17].

As mentioned above, medical grade honey dressings have been reported to be effective at treating chronic wounds of the lower extremities such as venous stasis ulcers and DFUs [5,9]. In the current study, we confirmed that the rate of reduction of wound surface area was greater in group B, whose wounds were treated with a honey-containing alginate dressing, than group A, whose wounds were treated with an alginate-only dressing (Fig. 1A). In addition, in the 1 week period immedi- ately after wound care, there was a greater reduction in the percentage of wound surface area in group $B$ than group A (Fig. 1B), indicating that honey had a beneficial effect when combined with alginate for the treatment of chronic wounds of the lower extremities. Medical grade honey-containing alginate dressing has been globally used for wound management with safety approved by US Food and Drug Administration in the United States and European Conformity in Europe. Factors desirable for promoting wound healing in dressing materials include antibiotic effects, antioxidant effects, anti-inflammatory effects, auto-debridement ability, and the ability to stimulate growth factor production. Honey-containing dressings are effective in wound healing because they have antibiotic, antioxidant, and anti-inflammatory effects. 
Medical grade honey has topical antimicrobial activity and can remove and inhibit the formation of biofilms, which is significant in the treatment of chronic wounds. Most honey-containing wound dressing materials contain medical grade Manuka honey with a $\mathrm{pH}$ of around 3.2-4.5. The low $\mathrm{pH}$ of medical grade honey provides an environment that inhibits the growth of bacteria. Moreover, honey has bactericidal effects against bacteria that easily colonize skin and wounds, such as P. aeruginosa, S. aureus, Escherichia coli, and Acinetobacter calcoaceticus $[18,19]$, as well as effectiveness against antibioticresistant bacteria $[20,21]$. These antibacterial properties have been attributed to the high sugar content of honey, its low $\mathrm{pH}$, and the production of hydrogen peroxide from honey which in turn creates free radicals [8-11]. In a recent study, methylglyoxal, which is a component of honey, was shown to damage bacteria flagella, inhibit mobility, and inactivate defensin-1, and was shown to be particularly effective at treating chronic wounds infected with methicillin-resistant $S$. aureus [20]. In the current study, there were no significant differences in the species that colonized most of the chronic wounds between the two groups (Table 2). Combination of the bacteriostatic effect of alginate and the bactericidal effect of honey combined with destruction and absorption of biofilm likely underlie the beneficial effects of the honey-containing alginate dressing. There were a significant number of wounds colonized with antibiotic-resistant bacteria in our study (18.75\% in group A and $23.81 \%$ in group B), suggesting that the medical grade honeycontaining alginate is effective against wounds colonized with antibiotic-resistant bacteria.

Medical grade honey can induce the removal of necrotic and contaminated tissue from wounds. The high concentration of sugars and high viscosity of honey form strong osmotic gradients that absorb fluid from the wound surface and surrounding subdermal tissue. The high osmotic pressure of Manuka honey, corresponding to $10^{5} \mathrm{~atm}$ of pressure, cause it to act as a hypertonic solution with excellent exudate absorption activity. This not only contributes to auto-debridement of the necrotic tissue, but also protects the wound surface from the external environment and bacteria due to formation of a hydration barrier until wound closure is established [22]. In this study, a large amount of exudate was recorded initially for most wounds. Considering that management of excessive exudate from chronic wounds is thought to be critical for the wound healing process, the excellent exudate absorption activity of medical grade honey creates an environment suitable for chronic wound closure. Moreover, by combining the strong exudate absorption capacity of honey with that of alginate, exudate removal ability is increased.

We recommend the use of a secondary dressing material with strong absorption capacity to prevent secondary infection from the wet wound surface due to the large amount of exudate present during the early phases of wound care. In this study, we used DryMax Foam as the secondary dressing material. DryMax is a superabsorbent dressing with a cellulose core and superabsorbent polymers contained within a polypropylene cover [23]. Use of superabsorbent dressings in patients with highly exudative wounds can prevent secondary wound infection, protect the perilesional skin from pathological changes caused by a prolonged inflammatory process, and keep the wound surface moist, contributing to wound healing [24] in a cost-effective manner. The large amount of exudate recorded in this study (50\% saturated wounds in group A and $47.62 \%$ in group B; $25 \%$ leaking wounds in group A and $28.57 \%$ in group B) based on the WUWHS assessment system [10] highlights how critical the management of exudate is for chronic wound healing. The use of superabsorbent dressings is therefore preferred in patients with highly exudative chronic wounds in our department. Combination of the strong exudate absorption capacity of honey with the superabsorbent properties of the secondary dressing is especially beneficial for patients who find it difficult to visit the hospital frequently for various reasons including lack of finances, because augmented absorption can reduce the need for frequent dressing changes, prolong the interval between hospital visits, and make outpatient-based wound care possible.

Lastly, medical grade honey has the ability to promote tissue repair following the release of acute inflammatory cytokines, fibroblasts, and revascularization. The mechanism of tissue repair is linked to the low $\mathrm{pH}$ and high glucose content of honey, which stimulate macrophages to secrete inflammatory cytokines, monocytes to secrete inflammatory mediators such as tissue necrosis factor, interleukin 6 , and interleukin 1 , and promotes fibroblast proliferation, contributing to tissue proliferation and remodeling [6]. In addition, honey is known to promote oxygen release from hemoglobin, which stimulates the proliferation of endothelial cells and vascular endothelial growth factor production, which promotes healing of wounds by improving tissue perfusion $[6,25]$. In this study, because the chronic wounds were mostly DFUs and venous stasis ulcers, both of which have underlying vascular causes, improving tissue perfusion in the periwound tissue and wound surface is important. 
Surgical reconstruction is usually the first choice for wound care of wounds with bone exposure, therefore we did not include these types of wounds in our study. However, if the wound base cannot be transformed sufficiently for surgical reconstruction using a local flap or skin graft, wound closure can fail, followed by infection and recurrence of chronic wounds. Moreover, if vascular conditions are unfavorable for free tissue transfer with insufficient surrounding tissue, surgical reconstruction as an option for treating lower extremity wounds is limited. Application of a honey-containing alginate dressing to this type of wound might allow development of a sufficiently healthy wound base for a secondary skin graft, and make surgery a feasible option with a reduction in the surgical failure rate. However, further studies are needed to test these hypotheses.

The limitation of this study is the study was designed as retrospective chart review and selection bias inevitably occurs. Therefore, the possibility that the various covariates suggested in this study influenced the treatment effects could not be completely excluded. However, it was suggested that difference between the two groups by covariate was not statistically significant by Mann-Whitney $U$ test and Fisher extraction. Moreover, to minimize the selection bias, the patients were reevaluated by propensity score matching resulting statistically significant results. After propensity score matching, the statistical power decreases from $<0.001$ to 0.021 , which is thought to be due to the effects of small number of patients, which is also a limitation of this study.

In conclusion, we found that treatment of chronic wounds of the lower extremities with honey-containing alginate dressing decreased wound area more rapidly than an alginate-only dressing as well as the duration until wound closure. Based on our results, application of a honey-containing alginate dressing for treating lower extremity chronic wounds, especially those infected with antibiotic-resistant bacteria, is a good treatment option.

\section{Conflict of interest}

No potential conflict of interest relevant to this article was reported.

\section{ORCID iDs}

Yeon Ji Lee

Kyeong Soo Park

Dong Yeon Kim https://orcid.org/0000-0002-8298-4036

https://orcid.org/0000-0001-6064-7947

https://orcid.org/0000-0001-6169-4518
Hyung-Sup Shim ～https://orcid.org/0000-0001-5156-2239

\section{Supplementary material}

Supplementary Fig. 1. After propensity score matching by nearest neighbor matching method, 13 pairs of patients from each group were matched.

Supplemental data can be found at: https://doi.org/10.22467/ jwmr.2021.01557

Supplementary Fig. 2. After propensity score matching, decreased wound area were re-evaluated resulting that reduction of wound area was greater in group $\mathrm{B}$ than in group $\mathrm{A}(\mathrm{P}=$ $0.201)$.

Supplemental data can be found at: https://doi.org/10.22467/ jwmr.2021.01557

\section{References}

1. Braddock M. Tissue repair and ulcer/wound healing: Institut Pasteur Euroconference. Molecular mechanisms, therapeutic targets and future directions. IDrugs 2005;8:381-3.

2. James GA, Swogger E, Wolcott R, et al. Biofilms in chronic wounds. Wound Repair Regen 2008;16:37-44.

3. Bowers S, Franco E. Chronic wounds: evaluation and management. Am Fam Physician 2020;101:159-66.

4. Gupta S, Andersen C, Black J, et al. Management of chronic wounds: diagnosis, preparation, treatment, and followup. Wounds 2017;29:S19-36.

5. Moghazy AM, Shams ME, Adly OA, et al. The clinical and cost effectiveness of bee honey dressing in the treatment of diabetic foot ulcers. Diabetes Res Clin Pract 2010;89:27681.

6. Minden-Birkenmaier BA, Bowlin GL. Honey-based templates in wound healing and tissue engineering. Bioengineering (Basel) 2018;5:46.

7. Lu J, Cokcetin NN, Burke CM, et al. Honey can inhibit and eliminate biofilms produced by Pseudomonas aeruginosa. Sci Rep 2019;9:18160.

8. Gethin G, Cowman S, Kolbach DN. Debridement for venous leg ulcers. Cochrane Database Syst Rev 2015;2015: CD008599.

9. Wang C, Guo M, Zhang N, et al. Effectiveness of honey dressing in the treatment of diabetic foot ulcers: a systematic review and meta-analysis. Complement Ther Clin Pract 2019;34:123-31. 
10. Davies P. Exudate assessment and management. Br J Community Nurs 2012;Suppl:S18-22.

11. Hartig SM. Basic image analysis and manipulation in ImageJ. Curr Protoc Mol Biol 2013; Chapter 14:Unit14.15.

12. Kane LT, Fang T, Galetta MS, et al. Propensity score matching: a statistical method. Clin Spine Surg 2020;33:120-2.

13. Aderibigbe BA, Buyana B. Alginate in wound dressings. Pharmaceutics 2018;10:42.

14. Coleman RJ, Lawrie G, Lambert LK, et al. Phosphorylation of alginate: synthesis, characterization, and evaluation of in vitro mineralization capacity. Biomacromolecules 2011; 12:889-97.

15. O’Meara S, Martyn-St James M, Adderley UJ. Alginate dressings for venous leg ulcers. Cochrane Database Syst Rev 2015;2015:CD010182.

16. Dumville JC, O’Meara S, Deshpande S, et al. Alginate dressings for healing diabetic foot ulcers. Cochrane Database Syst Rev 2013;2013:CD009110.

17. Mirzaei B, Etemadian S, Goli HR, et al. Construction and analysis of alginate-based honey hydrogel as an ointment to heal of rat burn wound related infections. Int J Burns Trauma 2018;8:88-97.

18. Blair SE, Cokcetin NN, Harry EJ, et al. The unusual antibacterial activity of medical-grade Leptospermum honey: antibacterial spectrum, resistance and transcriptome anal- ysis. Eur J Clin Microbiol Infect Dis 2009;28:1199-208.

19. Cooper RA, Jenkins L, Henriques AF, et al. Absence of bacterial resistance to medical-grade manuka honey. Eur J Clin Microbiol Infect Dis 2010;29:1237-41.

20. Henriques AF, Jenkins RE, Burton NF, et al. The effect of manuka honey on the structure of Pseudomonas aeruginosa. Eur J Clin Microbiol Infect Dis 2011;30:167-71.

21. Blaser G, Santos K, Bode U, et al. Effect of medical honey on wounds colonised or infected with MRSA. J Wound Care 2007;16:325-8.

22. Molan PC. Re-introducing honey in the management of wounds and ulcers: theory and practice. Ostomy Wound Manage 2002;48:28-40.

23. Stephen-Haynes J, Stephens C. Evaluation of a superabsorbent dressing in a primary care organization. Br J Community Nurs 2012;Suppl:S36.

24. Munter KC, De Lange S, Eberlein T, et al. Handling properties of a superabsorbent dressing in the management of patients with moderate-to-very high exuding wounds. J Wound Care 2018;27:246-53.

25. Majtan J, Bohova J, Garcia-Villalba R, et al. Fir honeydew honey flavonoids inhibit TNF- $\alpha$-induced MMP-9 expression in human keratinocytes: a new action of honey in wound healing. Arch Dermatol Res 2013;305:619-27. 